to the Review Group has recently been submitted.

The College continues to consider and consult about the development of continuing medical education.

The next Business Meeting will be held in the Council Room at the Royal College of Psy- chiatrists on Wednesday 23 March at $4.30 \mathrm{pm}$, after the Council Meeting. All members of the College are welcome to attend this meeting.

Professor CHRIS THOMPSON, Registrar, Royal College of Psychiatrists

\title{
Elections to the Fellowship, 1994
}

At the meeting of the Court of Electors held on 14 February 1994, the following applicants were approved.

\section{The Fellowship}

Dr F. E. A. Abdul Rahim, Dr M. F. S. Abdurahman, Dr P. Y. Abusah, Dr J. A. Baird, Dr R. C. Baldwin, Dr C. M. Bass, Dr C. V. R. Blacker, Dr D. R. Blackwood, Dr M. J. Bowman, Professor E. A. Bradley, Dr K. W. Bridges, Dr B. D. P. Browne, Dr J. Catalan, Dr R. N. Chitty, Dr D. A. G. Cook, Dr W. E. Dickson, Dr V. M. Evans, Dr R. Farmer, Dr U. Freeston, Dr K. W. M. Fulford, Dr P. Gaminiratne, Dr D. V. J. Harischandra, Dr M. L. Hartman, Dr N. M. Healy, Dr M. A. Hession, Dr C. C. Hunter, Professor J. A. Insua, Dr P. Jauhar, Dr D. V. Jones, Dr R. W. Jones, Dr S. H. Kamlana, Dr K. Kassim, Professor C. L. E. Katona, Dr N. Kaur, Dr D. I. Khoosal, Dr M. G. Livingston, Dr A. J. MacDonald, Dr E. S. Meltzer, Dr N. D. Minton, Dr P. B. Mitchell,
Dr D. H. Montgomery, Dr B. Moore, Dr A. D. Nikapota, Dr D. J. Nutt, Dr S. Olivieri, Dr K. Padamsee, Dr D. J. Pallis, Dr M R. Pokorny, Professor M. Sanati, Dr M. S. Shooter, Dr A. M. Silverman, Dr R. J. Simpson, Dr D. E. Somekh, Dr J. S. Strang, Dr A. G. Sutton, Dr M. P. Tam, Dr G. H. A. Tate, Dr C. C. Tennant, Dr P. L. Thakur, Dr M. J. J. Thompson, Dr S. W. Turner, Dr M. Tweddle, Dr E. A. Vizard, Dr D. R. D. Wallace, Dr A. G. C. Wijeyeratne, Dr D. G. Wilkinson, Dr A. H. Yonace, Dr. H. A. M. Youssef and Dr A. S. Zigmond.

\section{Membership under Bye-Law III 2 (ii)}

Dr S. Chawla, Professor M. S. Farooqi, Dr J. A. Fernandez, Dr M. E. Heti-Davies, Professor O. Morakinyo, Dr R. E. Steel and Professor K. Yamazaki.

\section{New Associate}

Dr J. M. Atkinson. 